\title{
ON PINCHING DEFORMATIONS OF RATIONAL MAPS
}

\author{
BY LEI TAN
}

\begin{abstract}
We introduce the notion of the dynamical length of an invariant arc of a rational map $R$. A pinching deformation is a sequence of topological deformations of $R$ such that the corresponding dynamical length shrinks to zero. We show that if the sequence converges to a rational map then the spherical diameter of the corresponding arc also shrinks to zero. We use this result to show that if the grand orbits of the closure of finitely many such arcs separate the Julia set, the deformations of $R$ diverge. This is a generalization of a result stated by P. Makienko but with a different approach. We also present a rich collection of examples.

(c) 2002 Éditions scientifiques et médicales Elsevier SAS
\end{abstract}

RÉSUMÉ. - Nous introduisons la notion de longueur dynamique d'un arc invariant par une fraction rationnelle $R$. Une déformation pincée est une suite de déformations topologiques de $R$ qui contracte les longueurs dynamiques correspondantes jusqu'à zéro. On montre que si la suite converge à une fraction rationnelle, le diamètre sphérique des arcs correspondants tend également vers zéro. Nous utilisons ce résultat pour montrer que, si l'orbite inverse de l'adhérence d'un nombre fini de tels arcs sépare l'ensemble de Julia, la suite des déformations diverge. Ceci est une forme plus générale d'un résultat énoncé par P. Makienko, mais avec une approche différente. Nous présentons également une large classe d'exemples divers.

(C) 2002 Éditions scientifiques et médicales Elsevier SAS

\section{Introduction}

Pinching deformations for rational maps were first introduced by P. Makienko from the analogy of Kleinian groups. A recent paper of his [5] states a result (part of Theorem A) about divergence of such deformations, together with many interesting applications. This result, however, relies on two intermediate statements, the proofs of which seem incomplete (see Appendix A below). We present here a generalization of it, from a different approach. Our method is based upon C. Petersen proof of a similar result [9].

We start with a

Definition (Dynamical length of an invariant set). - For a map $R$, we say that a set $\gamma$ is $R$-invariant if $R(\gamma) \subset \gamma$. If furthermore $\gamma \subset V \subset \overline{\mathbb{C}}$, with $V$ a hyperbolic open set, we define the dynamical length $l_{V}(\gamma, R)$ relative to $V$ to be

$$
l_{V}(\gamma, R)=\sup _{z \in \gamma} d_{V}(z, R(z))
$$

where $d_{V}$ denotes the hyperbolic metric on $V$. Note that if $V^{\prime} \supset V, l_{V^{\prime}}(\gamma, R) \leqslant l_{V}(\gamma, R)$ by the Schwarz Lemma. For explicit examples see the next definition. 
Denote by diam $_{\sigma}$ the spherical diameter. In the following we will always assume that $\gamma$ is connected. One of our central topics here is to relate $l_{V}(\gamma, R)$ to $\operatorname{diam}_{\sigma}(\bar{\gamma})$. Roughly speaking, we will show that for $R$ a degree $>1$ rational map and $R_{n}$ a sequence of rational maps topologically conjugate to $R$ with $h_{n}$ as conjugacy such that $l_{h_{n}(V)}\left(h_{n}(\gamma), R_{n}\right) \rightarrow 0$, if $R_{n}$ converges uniformly then $\operatorname{diam}_{\sigma}\left(\overline{h_{n}(\gamma)}\right) \rightarrow 0$. We will start with a concrete construction of such pairs $\left(h_{n}, R_{n}\right)$, namely pinching deformations of $R$. Our definition is slightly more general than that of Makienko [5].

Definition (The model system). - For $l>0$, let $B_{l}$ denote the horizontal strip $\{x+i y \mid$ $\left.|y|<\frac{\pi}{2 l}\right\}$, and $T_{\sigma}$ denote the translation $z \mapsto z+\sigma$. We consider the couple $\left(B_{l}, T_{\sigma}\right)$ as a model dynamical system, with $\mathbb{R}$ as the central line. For the system $\left(B_{l_{0}}, T_{1}\right)$, and for any $z \in \mathbb{R}$, the distance $d_{B_{l_{0}}}\left(z, T_{1}(z)\right)$ coincides with the hyperbolic length of the segment $\left[z, T_{1}(z)\right]$, and is exactly $l_{0}$ (see for example McMullen [6], p. 12). Therefore $l_{B_{l_{0}}}\left(\mathbb{R}, T_{1}\right)=l_{0}$. More generally $l_{B_{l_{0}}}\left(\mathbb{R}, T_{l / l_{0}}\right)=l$.

DEFINITION (The pinching model). - For $l_{0}>0$ and $t \in\left[0,1\right.$ [, let $t \mapsto l_{t}$ be a decreasing continuous function tending to 0 as $t \rightarrow 1^{-}$, and choose a quasi-conformal map $S_{t}: B_{l_{0}} \rightarrow B_{l_{0}}$ such that $S_{t}(z)$ is a $C^{1}$-function of $(t, z), S_{t}(\mathbb{R})=\mathbb{R}$ and $S_{t}$ conjugates $\left(B_{l_{0}}, T_{1}\right)$ to $\left(B_{l_{0}}, T_{l_{t} / l_{0}}\right)$. As $t \rightarrow 1^{-}, l_{t}=l_{B_{l_{0}}}\left(\mathbb{R}, T_{l / l_{0}}\right) \rightarrow 0$ and the quasi-conformal constant of $S_{t}$ tends to $\infty$.

(Although the concrete form of $S_{t}(z)$ is not relevant for the purpose of this work, one should keep in mind that a more careful choice of it may be important for further studies.)

Definition (Admissible pair). - Let $R: \overline{\mathbb{C}} \rightarrow \overline{\mathbb{C}}$ be a rational map and $k \in \mathbb{N}$. An open arc $\gamma \subset \overline{\mathbb{C}}$ (i.e. a continuous and injective image of the segment $(0,1))$ together with a neighborhood $U$ of it, is called $R^{k}$-admissible, if:

1. $R^{k}(\gamma)=\gamma, R^{k}(U)=U,\left.R^{k}\right|_{U}$ is univalent and $U, R(U), \ldots, R^{k-1}(U)$ are mutually disjoint (note that $U$ cannot contain critical points but may contain points in their forward orbits).

2 . There is a conformal normalization $\Phi:\left(U, \gamma, R^{k}\right) \rightarrow\left(B_{l_{0}}, \mathbb{R}, T_{1}\right)$ for some $l_{0}>0$, in other words there exists an analytic homeomorphism $\Phi: U \rightarrow B_{l_{0}}$ mapping $\gamma$ onto $\mathbb{R}$ and satisfying $\left.\Phi \circ R^{k}\right|_{U}=\left.T_{1} \circ \Phi\right|_{U}$.

Thus $U$ is an $R^{k}$-invariant strip with $\gamma$ as the central line. We have $l_{U}\left(\gamma, R^{k}\right)=l_{0}$, and it coincides with the hyperbolic length (relative to $U$ ) of the sub-arc in $\gamma$ between a point $z$ and $R^{k}(z)$.

Examples of admissible pairs can be obtained, as in [5], by lifting suitable geodesics with collar neighborhoods in the quotient Riemann surface of $R$, or, as in [9], by projecting lines and strips in the logarithmic linearizing coordinates of an attracting point, or in the Fatou coordinate of a parabolic point. Details and further examples can be found in $\$ 5-7$ below, including an example with $\gamma$ containing postcritical points.

A more concrete example can be given for the map $z^{2}+c$ with $0<c<1 / 4$, with $\gamma$ the real segment between the two fixed points, and $U$ a suitable neighborhood of $\gamma$, symmetric with respect to $\mathbb{R}$.

Definition (Pinching). - Let $(\gamma, U)$ be an $R^{k}$-admissible pair. Fix a choice of $S_{t}$ on $B_{l_{0}}$. Denote by $\left.E_{t}\right|_{U}$ the pulled back ellipse field of the circle field on $B_{l_{0}}$ by $S_{t} \circ \Phi$. With the help of $R$ it generates an invariant ellipse field $E_{t}$ of $R$, conformal outside the grand orbit of $U$. Choose a quasi-conformal map $\varphi_{t}:\left(\overline{\mathbb{C}}, E_{t}\right) \rightarrow \overline{\mathbb{C}}$ integrating $E_{t}$ (given by Ahlfors-Bers Theorem), and set $R_{t}=\varphi_{t} \circ R \circ \varphi_{t}^{-1}$. We call $\left(\varphi_{t}, R_{t}\right)_{t \in[0,1)}$ a path of pinching deformations of $R$ along $(\gamma, U)$. Note that if $\varphi_{t}$ is replaced by $H_{t} \circ \varphi_{t}$, with $H_{t}$ a Möbius transformation, then $R_{t}$ is replaced by $H_{t} \circ R_{t} \circ H_{t}^{-1}$. 
Remark 1. - For a path of pinching deformations the pair $\left(\gamma_{t}, U_{t}\right):=\left(\varphi_{t}(\gamma), \varphi_{t}(U)\right)$ is $R_{t}^{k}$-admissible and $\varphi_{t}$ shrinks the corresponding dynamical length to 0 , in other words

$$
l_{U_{t}}\left(\gamma_{t}, R_{t}^{k}\right)=l_{t} \rightarrow 0 \quad \text { as } t \rightarrow 1 .
$$

This is because the composition of the following mappings $U_{t} \stackrel{\varphi_{t}^{-1}}{\longrightarrow} U \stackrel{\Phi}{\longrightarrow} B_{l_{0}} \stackrel{S_{t}}{\longrightarrow} B_{l_{0}}$ is conformal, mapping $\gamma_{t}$ to $\mathbb{R}$ and conjugating $R_{t}^{k}$ to $T_{l_{t} / l_{0}}$. As $U$ is contained in the Fatou set $F(R)$, we have also $l_{F\left(R_{t}\right)}\left(\gamma_{t}, R_{t}^{k}\right) \rightarrow 0$. Moreover, for any fixed $t$, the dynamical length $l_{U_{t}}\left(\gamma_{t}, R_{t}^{k}\right)$ remains unchanged if the couple $\left(\varphi_{t}, R_{t}\right)$ is replaced by $\left(H_{t} \circ \varphi_{t}, H_{t} \circ R_{t} \circ H_{t}^{-1}\right)$.

DEFINITION (Pinching deformations along an admissible set). - More generally, we call an admissible set for a rational map $R$, a collection of finitely many admissible pairs $\left\{\left(\gamma^{i}, U_{i}\right)\right.$, $i=1, \ldots, \nu\}$ such that the orbits of distinct $U_{i}$ 's are disjoint. We can then define on each $U_{i}$ the pulled back ellipse field by the corresponding $S_{i, t} \circ \Phi_{i}$ (for some choice of $S_{i, t}$ ), and then an $R$-invariant ellipse field. As above, this will generate, for each choice of the integrating map $\varphi_{t}$, a path of pinching deformations $\left(\varphi_{t}, R_{t}\right)$ along $\left\{\left(\gamma^{i}, U_{i}\right)\right\}$.

We want to study the behavior of $R_{t}$ as $t \rightarrow 1$, in particular the following questions:

1 (convergence). When does a subsequence of $R_{t}$ converge uniformly? How about $\varphi_{t}$ ?

$1^{\prime}$ (path convergence). When does the entire path $R_{t}$ converge uniformly?

Denote by $\rightrightarrows$ the uniform convergence on $\overline{\mathbb{C}}$.

2 (consequence of convergence). In case $R_{t_{n}} \rightrightarrows G$ for some $t_{n} \rightarrow 1$, what can we say about the limit of $\varphi_{t_{n}}(\gamma)$ and the dynamics of $G$ ?

3 (divergence). When does no sequence of $H_{t} \circ R_{t} \circ H_{t}^{-1}$ converge uniformly, no matter which Möbius maps $H_{t}$ are chosen?

Denote by $\mathcal{U}_{d}$ the space of rational maps of degree $d$ with the topology of uniform convergence on $\overline{\mathbb{C}}$, and by $\mathcal{V}_{d}$ the quotient topological space of $\mathcal{U}_{d}$ under Möbius conjugation. Let $\pi: \mathcal{U}_{d} \rightarrow \mathcal{V}_{d}$ be the canonical projection. For a family $F_{t} \in \mathcal{U}_{d}$, we say that $\pi\left(F_{t}\right) \rightarrow \infty$ if $\pi\left(F_{t}\right)$ has no convergent sequences. Question 3 can be then reformulated as follows:

$3^{\prime}$ (divergence). When does $\pi\left(R_{t}\right) \rightarrow \infty$ as $t \rightarrow 1$ ?

In this paper we will mainly study questions 2 and 3, following Makienko (but in a more general setting). Question 2 will be relevant in answering question 3 with an argument by contradiction. For papers treating Question 1, see Cui [2,3] and Haïssinsky [4].

For $A, B \subset \overline{\mathbb{C}}$, we say that $A$ separates $B$ if $B$ intersects more than one component of $\overline{\mathbb{C}} \backslash A$. So if $A$ does not separate $B$, then $B$ is contained in the union of $A$ together with exactly one component of $\overline{\mathbb{C}} \backslash A$. Recall that $\operatorname{diam}_{\sigma}$ denotes the spherical diameter. Denote by $F(R)$ the Fatou set of $R$. Our main result is:

THEOREM A. - Let $R$ be a rational map of degree $\geqslant 2$, with $\left\{\left(\gamma^{i}, U_{i}\right), i=1, \ldots, \nu\right\}$ an admissible set and $\gamma^{i}$ of period $k_{i}$. Set $\Gamma_{0}=\bigcup_{i} \bigcup_{m \geqslant 0} R^{m}\left(\overline{\gamma^{i}}\right)$ and $\Gamma_{j}=R^{-j}\left(\Gamma_{0}\right)$. Assume that $\left(\varphi_{t}, R_{t}\right)$ is a path of pinching deformations of $R$ along this admissible set (in particular $\varphi_{t}$ is a topological conjugacy from $R$ to $R_{t}$ and $l_{\varphi_{t}\left(U_{i}\right)}\left(\varphi_{t}\left(\gamma^{i}\right), R_{t}^{k_{i}}\right) \rightarrow 0$ (as $\left.t \rightarrow 1\right)$ for each $\left.i\right)$. More generally assume that $\varphi_{t}$ are topological conjugacies from $R$ to rational maps $R_{t}$ such that $l_{F\left(R_{t}\right)}\left(\varphi_{t}\left(\gamma^{i}\right), R_{t}^{k_{i}}\right) \rightarrow 0$ (as $\left.t \rightarrow 1\right)$ for each $i$.

(a) Assume $R_{t_{n}} \rightrightarrows G$ for some sequence $t_{n} \rightarrow 1$. Then

1. For any $j \geqslant 0$ and any connected component $\Gamma$ of $\Gamma_{j}$, we have diam $m_{\sigma} \varphi_{t_{n}}(\Gamma) \rightarrow 0$ as $n \rightarrow \infty$. Moreover if $\Gamma$ is a component of $\Gamma_{0}$, the sequence $\varphi_{t_{n}}(\Gamma)$ splits into at most finitely many subsequences, each converging to a parabolic periodic point of $G$.

2. For every $j \geqslant 0$, there is exactly one component $\Delta_{j}$ of $\overline{\mathbb{C}} \backslash \Gamma_{j}$ intersecting $J(R)$. For any other component $\Delta_{j}^{\prime}$ of $\overline{\mathbb{C}} \backslash \Gamma_{j}$, diam ${ }_{\sigma} \varphi_{t_{n}}\left(\overline{\Delta_{j}^{\prime}}\right) \rightarrow 0$ as $t_{n} \rightarrow 1$. For $j=0$, and 
any component $\Delta_{0}^{\prime}$ of $\overline{\mathbb{C}} \backslash \Gamma_{0}$ distinct from $\Delta_{0}$, the sequence $\varphi_{t_{n}}\left(\overline{\Delta_{0}^{\prime}}\right)$ splits into at most finitely many subsequences, each converging to a parabolic periodic point of $G$. Moreover all critical points are contained in $\Delta_{0}$.

(b) If $\Gamma_{j}$ separates $J(R)$ for some $j \geqslant 0$, or $\Gamma_{0}$ separates the critical points, then $\pi\left(R_{t}\right) \rightarrow \infty$ as $t \rightarrow 1$.

Part (b) generalizes Theorem A.(2) in [5]. Examples realizing different cases can be found in $\$ 7$ below. Under the extra assumption that $R$ is geometrically finite, Cui announced a result in [3] which can be considered as the converse of (b), namely if $\Gamma_{j}$ does not separate $J(R)$ for all $j \geqslant 0$, then for some suitable choice of pinching deformations, both paths $R_{t}$ and $\varphi_{t}$ converge uniformly to $G$ and $\varphi_{1}$ respectively, and $G \circ \varphi_{1}=\varphi_{1} \circ R$.

The proof of our theorem is in fact a soft argument, and may lead to various side results with weaker assumptions. We will mention three of them in the paper: Proposition B drops off the rationality of $R_{t}$, Propositions $\mathrm{B}$ and $\mathrm{C}$ do not require the existence of topological conjugacies and Propositions $\mathrm{C}$ and $\mathrm{D}$ replace the exact forward invariance assumption of $\gamma^{i}$ by the weak forward invariance (i.e., $\left.R^{k_{i}}\left(\gamma^{i}\right) \subset \gamma^{i}\right)$.

In the following, we will split Theorem A into several independent parts: Part (a). 1 will follow from Lemma 2.1, (a).2 is proved in $\S 3$, and (b) in $\S 4$. Propositions B and C will be stated and proved in $\S 2$. Proposition D is in $\S 3$. In $\S 6$ we will give interpretations of our results in terms of Teichmüller theory of rational maps (Theorem E and Corollary F). $\$ 7$ contains many relevant examples. Impatient readers can read directly Lemmas 2.1 and 3.1. The rest follows more or less naturally.

\section{Consequence of convergence, I}

The following simple facts will be used frequently and implicitly: If a sequence of continuous maps $F_{n}: \overline{\mathbb{C}} \rightarrow \overline{\mathbb{C}}$ converges uniformly to a map $G$, then for any convergent sequence $z_{n} \rightarrow z_{\infty}$ (of points or non-empty compact sets in the Hausdorff topology), we have $\lim F_{n}\left(z_{n}\right)=G\left(z_{\infty}\right)$. If furthermore $F_{n}=R_{n}$ are rational maps of constant degree then $G$ is a rational map of the same degree, and $R_{n}^{m} \rightrightarrows G^{m}$ for any fixed integer $m>0$. If moreover $z_{n}=h_{n}(z)$, where $h_{n}$ is a topological conjugacy from $R_{0}$ to $R_{n}$ then $z_{\infty}$ is fixed by $G$ whenever $z$ is fixed by $R_{0}$.

LEMMA 2.1. - Let $R$ be a rational map of degree $d \geqslant 2$ and $(\gamma, U)$ be an $R^{k}$-admissible pair. Let $R_{n}$ be a sequence of rational maps topologically conjugate to $R$ with $h_{n}$ as conjugacies. Assume that $l_{h_{n}(U)}\left(h_{n}(\gamma), R_{n}^{k}\right) \rightarrow 0$ and $R_{n} \rightrightarrows G$. Then

$$
\lim _{n \rightarrow \infty} \operatorname{diam}_{\sigma} h_{n}(\bar{\gamma})=0
$$

Moreover the sequence $h_{n}(\bar{\gamma})$ splits into at most finitely many subsequences, each converging to a parabolic periodic point of $G$.

Proof. - The idea is to adopt Petersen's proof in [9] (of his Proposition 4.1 and 4.3) to a more general setting.

Using the classification of periodic Fatou components one can easily show that $U$ is contained in the immediate attracting basin $\Lambda$ of a periodic point $\alpha$, which is either attracting (non-superattracting) or parabolic.

The period of $\alpha$ divides $k$ (may be equal). The period of $\Lambda$ is equal to $k$ in the parabolic case with $\left(R^{k}\right)^{\prime}(\alpha)=1$, and is equal to the period of $\alpha$ otherwise.

To simplify the notation set $f=R^{k}, f_{n}=R_{n}^{k}$ and $g=G^{k}$, so $f_{n} \rightrightarrows g$. Set also $\gamma_{n}=h_{n}(\gamma)$, $U_{n}=h_{n}(U), \alpha_{n}=h_{n}(\alpha)$ and $\Lambda_{n}=h_{n}(\Lambda)$ etc. 
To obtain a contradiction, suppose $\lim \sup _{n \rightarrow \infty} \operatorname{diam}_{\sigma} \overline{\gamma_{n}}>0$.

Step 1 . Taking a few subsequences if necessary we may assume also:

$\alpha_{n} \rightarrow \alpha_{\infty} \in \overline{\mathbb{C}}, \overline{\gamma_{n}} \rightarrow Y_{\infty}$ in the Hausdorff topology on compact sets, and $\operatorname{diam}_{\sigma}\left(Y_{\infty}\right)>0$. As $\gamma_{n}$ is connected, $Y_{\infty}$ is a continuum (compact connected).

Replacing $f_{n}$ by $H \circ f_{n} \circ H^{-1}$ and $g$ by $H \circ g \circ H^{-1}$ if necessary, where $H$ is a Möbius map sending $\alpha_{\infty}$ to 0 , we may assume $\alpha_{\infty}=0$.

Step 2. We claim that $g(0)=0, g^{\prime}(0)=1$ : As $f_{n}$ fixes $\alpha_{n}$ and $\alpha_{n} \rightarrow 0$, we have $g(0)=0$. Furthermore, if $\alpha$ is parabolic we have $f_{n}^{\prime}\left(\alpha_{n}\right) \equiv 1$; if $\alpha$ is attracting, the Maskit inequality (see Lemma 5.1 below and the subsequent remark) gives $f_{n}^{\prime}\left(\alpha_{n}\right) \rightarrow 1$. In both cases $g^{\prime}(0)=1$. This implies $g$, therefore $f_{n}$ for large $n$, are univalent on a fixed neighborhood $\bar{D}_{r}$ of 0 .

Step 3. Normalization so that $U_{n} \subset \mathbb{C}^{*}$. Let $z_{n} \in \partial \Lambda_{n}$ be a point closest to $\infty$. We claim that $\left|z_{n}\right| \geqslant r$, for otherwise $\partial \Lambda_{n} \subset D_{r}$ but $f_{n}$ is not injective on any neighborhood of $\partial \Lambda_{n}$.

Taking again a subsequence if necessary, we may assume $z_{n} \rightarrow z_{\infty} \neq 0$. Set $H_{n}(z)=\frac{z-\alpha_{n}}{1-z / z_{n}}$ if $z_{n} \neq \infty$ and $H_{n}(z)=z-a_{n}$ otherwise, set $H_{\infty}(z)=\frac{z}{1-z / z_{\infty}}$ if $z_{\infty} \neq \infty$ and $H_{\infty}(z)=z$ otherwise. In any case $H_{n}\left(\alpha_{n}\right)=0, H_{n}\left(z_{n}\right)=\infty$ and $H_{n} \rightrightarrows H_{\infty}$.

Conjugating $f_{n}$ by $H_{n}$ and $g$ by $H_{\infty}$ if necessary, we may assume $\alpha_{n}=0$ and $z_{n}=\infty$. In particular $U_{n} \subset \Lambda_{n} \backslash\left\{\alpha_{n}\right\} \subset \mathbb{C}^{*}$.

Step 4. Inequalities. We will use the following basic inequality (see for example Milnor, [8], Appendix A): For any simply connected domain $V \subset \mathbb{C}^{*}$, any $z \in V$, and for $\lambda_{V}(z)$ the coefficient function of the hyperbolic metric on $V$, we have:

$$
\lambda_{V}(z) \geqslant \frac{1}{2 d_{\mathbb{C}}(z, \partial V)} \geqslant \frac{1}{2|z|} .
$$

Set $\eta(z)=\frac{1}{2|z|}$. Let $d_{\eta}$ denote the hyperbolic metric on $\mathbb{C}^{*}$ with $\eta$ as coefficient function. Then for any arc $\kappa \subset V \subset \mathbb{C}^{*}$, we have length ${ }_{\eta}(\kappa) \leqslant$ length $_{V}(\kappa)$.

Claim. - For any $n$ and any $z \in \gamma_{n}$, we have $d_{\eta}\left(f_{n}(z), z\right) \leqslant l_{U_{n}}\left(\gamma_{n}, f_{n}\right)$.

Proof. - Let $\kappa$ be the sub-arc of $\gamma_{n}$ between $f_{n}(z)$ and $z$. Then

$$
d_{\eta}\left(f_{n}(z), z\right) \leqslant \text { length }_{\eta}(\kappa) \leqslant \text { length }_{U_{n}}(\kappa)=l_{U_{n}}\left(\gamma_{n}, f_{n}\right)
$$

By assumption $l_{U_{n}}\left(\gamma_{n}, f_{n}\right) \rightarrow 0$ as $n \rightarrow \infty$.

Step 5. Contradiction. Note that $f_{n} \rightrightarrows g$. Let $x \in\left(Y_{\infty} \cap \bar{D}_{r}\right) \backslash\{0\}$ such that $g(x) \neq \infty$. Choose a sequence $x_{n}^{\prime} \in \overline{\gamma_{n}}$ and then $x_{n} \in \gamma_{n}$ such that $x_{n} \rightarrow x$ as $n \rightarrow \infty$. Then

$$
d_{\eta}(g(x), x) \leqslant d_{\eta}\left(g(x), g\left(x_{n}\right)\right)+d_{\eta}\left(g\left(x_{n}\right), f_{n}\left(x_{n}\right)\right)+d_{\eta}\left(f_{n}\left(x_{n}\right), x_{n}\right)+d_{\eta}\left(x_{n}, x\right) .
$$

The right hand side converges to 0 as $n \rightarrow \infty$, since $g$ is continuous, $f_{n} \rightrightarrows g$,

$$
d_{\eta}\left(f_{n}\left(x_{n}\right), x_{n}\right) \leqslant l_{U_{n}}\left(\gamma_{n}, f_{n}\right) \rightarrow 0
$$

Thus $g(x)=x$. Hence $g(z)=z$ for all $z \in\left(Y_{\infty} \cap \bar{D}_{r}\right) \backslash\{0\}$. This implies that $g$ is the identity map as $Y_{\infty} \cap \bar{D}_{r}$ is a non-empty continuum. But $g=G^{k}$ is a rational map of degree $>1$. A contradiction.

The following statement stresses the essence of the above result and drops off irrelevant assumptions such as the rationality of $R$ and $R_{n}$ and the topological conjugations between them. 
PROPOSITION B. - Let $F_{n}$ be any sequence of holomorphic maps, each defined on a proper attracting basin $\Lambda_{n}$ of degree $d_{n} \geqslant 2$ of an attracting or parabolic fixed point $\alpha_{n}$, and with $\left(\gamma_{n}, U_{n}\right)$ an $F_{n}^{q}$-admissible pair in $\Lambda_{n}$. Assume $\alpha_{n} \rightarrow \alpha_{\infty}$. Then the following four properties cannot all hold at the same time:

1. $l_{U_{n}}\left(\gamma_{n}, F_{n}^{q}\right) \rightarrow 0$.

2. $\left.\lim \sup \operatorname{diam}_{\sigma}\left(\overline{\gamma_{n}}\right)\right)>0$.

3. $F_{n}$ converges uniformly on some neighborhood of $\alpha_{\infty}$ to a map $G$.

4. $G^{q} \neq i d$.

Proof. - Set $f_{n}=F_{n}^{q}, g=G^{q}$. Assume that all four conditions of the proposition are satisfied. Then Steps $1-5$ above lead to a contradiction.

Examples satisfying 1,2 and 4 are trivial to construct, satisfying 1,2 and 3 can be constructed via normalization, and 2, 3 and 4 can be constructed by setting $F_{n} \equiv G$ for a suitable $G$. Our pinching deformations provide examples satisfying 1,3 and 4 , and the conclusion is that 2 is not satisfied.

We state here a result whose proof is classical (see for example Petersen [9], Lemma 3.3):

LEMMA 2.2. - For $(\gamma, U)$ an admissible pair, the closure of $\gamma$ is equal to $\gamma \cup\{\alpha\} \cup\{\beta\}$, with $\alpha$ as the limit of any forward orbit in $\gamma$ and an attracting (non-superattracting) or parabolic point; and $\beta$ as the limit of any backward orbit in $\gamma$ and a periodic point in $J(R)$.

(With the help of the Snail Lemma one can also show that $\beta$ is either repelling or parabolic. But we don't need the result here.)

The following is another variation of Lemma 2.1. Again, the topological conjugations are not required, nor the exact forward invariance of admissible pairs.

Proposition C. - For $d \geqslant 2$ and $k>0$, assume that $R_{n}$ is a sequence of rational maps of degree d each having an $R_{n}^{k}$-invariant connected set $\gamma_{n}$ (i.e., $\left.R_{n}^{k}\left(\gamma_{n}\right) \subset \gamma_{n}\right)$, satisfying:

(1) $\gamma_{n} \subset F\left(R_{n}\right)$ and $l_{F\left(R_{n}\right)}\left(\gamma_{n}, R_{n}^{k}\right) \rightarrow 0$ as $n \rightarrow \infty\left(\right.$ or $\gamma_{n} \subset \overline{\mathbb{C}} \backslash\left\{a_{n}, b_{n}, c_{n}\right\}$,

$$
\inf \left\{d_{\sigma}\left(a_{n}, b_{n}\right), d_{\sigma}\left(b_{n}, c_{n}\right), d_{\sigma}\left(c_{n}, a_{n}\right) \mid n \in \mathbb{N}\right\}>0
$$

and $\left.l_{\overline{\mathbb{C}}_{\backslash}\left\{a_{n}, b_{n}, c_{n}\right\}}\left(\gamma_{n}, R_{n}^{k}\right) \rightarrow 0\right)$;

(2) $R_{n}$ converges uniformly.

Then $\operatorname{diam}_{\sigma}\left(\overline{\gamma_{n}}\right) \rightarrow 0$. Moreover, if for each $n, \gamma_{n}$ together with some neighborhood $U_{n}$ forms an admissible pair, and $\overline{\gamma_{n}}$ contains an attracting periodic point, then $\gamma_{n}$ accumulates to parabolic points of $G$ as $n \rightarrow \infty$.

As an example, take $R_{n} \equiv R, \gamma_{0}$ a compact connected invariant set contained in the immediate basin $\Lambda$ of an (super)-attracting fixed point and $\gamma_{n}=R^{n}\left(\gamma_{0}\right)$. By Schwarz' Lemma $\left.R\right|_{\gamma_{0}}$ is a strong contraction with respect to the hyperbolic metric on $\Lambda$. From this it is easy to show $l_{F(R)}\left(\gamma_{n}, R\right) \rightarrow 0$. And, of course, $\operatorname{diam}_{\sigma}\left(\overline{\gamma_{n}}\right) \rightarrow 0$.

Proof of Proposition C (note the Maskit inequality is not needed). - The idea to show $\operatorname{diam}_{\sigma}\left(\overline{\gamma_{n}}\right) \rightarrow 0$ is very similar. So we will only give a sketch. Again set $f_{n}=R_{n}^{k}$ and $g=\lim f_{n}$. Skip Step 2 above. In Step 3 perturb three repelling periodic points of $g$ and then normalize $f_{n}$ so that $F\left(f_{n}\right) \subset C \backslash\{0,1\}$. In Steps 4 and 5 replace the two-point-metric $d_{\eta}$ by the threepoint-(hyperbolic)-metric $d_{\mathbb{C} \backslash\{0,1\}}$ and then use the inequality $d_{\mathbb{C} \backslash\{0,1\}} \leqslant d_{F\left(f_{n}\right)}$ coming from Schwarz' Lemma. (This idea was pointed out to me by C. McMullen.)

Now we show that $\gamma_{n}$ accumulates to parabolic points, under the extra assumption that $\gamma_{n}$ is part of an admissible pair and $\overline{\gamma_{n}}$ contains an attracting point $\alpha_{n}$. By Lemma 2.2, 
$\overline{\gamma_{n}}=\gamma_{n} \cup\left\{\alpha_{n}\right\} \cup\left\{\beta_{n}\right\}$ with $\beta_{n}$ in the Julia set. As $\alpha_{n}$ is attracting, $\alpha_{n} \neq \beta_{n}$. Now assume $\overline{\gamma_{n_{i}}} \rightarrow\{x\}$ for some subsequence. Then $\alpha_{n_{i}} \rightarrow x$ and $\beta_{n_{i}} \rightarrow x$. Therefore $x$ is a multiple fixed point of $G^{k}$. So $\left(G^{k}\right)^{\prime}(x)=1$.

Proof of Theorem A. Part (a).1. - Assume at first that $\Gamma$ is a component of

$$
\Gamma_{0}=\bigcup_{i=1}^{\nu} \bigcup_{m \geqslant 0} R^{m}\left(\overline{\gamma^{i}}\right) .
$$

As each $\gamma^{i}$ is periodic for $R$, the set $\Gamma$ is the union of finitely many elements in $\left\{R^{m}\left(\overline{\gamma^{i}}\right)\right\}_{i, m}$. To obtain a contradiction we assume $\lim \sup \operatorname{diam}_{\sigma}\left(\varphi_{t_{n}}(\Gamma)\right)>0$. Then there are $i, m$ and $n_{k}$ such that $R^{m}\left(\overline{\gamma^{i}}\right)$ has definite spherical diameter under the actions of $\varphi_{t_{n_{k}}}$. As $\left(R^{m}\left(\gamma^{i}\right), R^{m}\left(U_{i}\right)\right)$ is an admissible pair, we can apply Lemma 2.1 or Proposition $C$ and get a contradiction.

Now let $\Gamma^{\prime}$ be a connected component of $\Gamma_{j}=R^{-j}\left(\Gamma_{0}\right)$. If its diameter under the actions of $\varphi_{t_{n}}$ does not tend to 0 , we may assume (taking a few subsequences if necessary) $\varphi_{t_{n}}\left(\Gamma^{\prime}\right) \rightarrow \Gamma_{\infty}^{\prime}, \operatorname{diam}_{\sigma} \Gamma_{\infty}^{\prime}>0$ and $\varphi_{t_{n}}(\Gamma) \rightarrow \Gamma_{\infty}$. But $G^{j}\left(\Gamma_{\infty}^{\prime}\right)=\Gamma_{\infty}$ (where $G$ is the uniform limit of $R_{t_{n}}$ ) and $\Gamma_{\infty}$ is a point by above. This is not possible.

Now let $\alpha^{i}$ be the attracting or parabolic point in $\overline{\gamma^{i}}$ (by Lemma 2.2). Then either $\varphi_{t_{n}}\left(\alpha^{i}\right)$ are all attracting, or all parabolic with constant multiplier. Applying Lemma 2.1 or Proposition C we conclude that $\varphi_{t_{n}}(\Gamma)$ accumulates to parabolic points of $G$, for any component $\Gamma$ of $\Gamma_{0}$.

\section{Consequence of convergence, II}

LEMMA 3.1. - Let $R$ be a rational map of degree $d \geqslant 2$ and $h_{n}$ a sequence of homeomorphisms such that $R_{n}:=h_{n} \circ R \circ h_{n}^{-1}$ are rational maps. Assume $R_{n} \rightrightarrows G$. Then for any open set $D$ containing points of $J(R)$ and having a connected closure, lim inf $\operatorname{diam}_{\sigma} h_{n}(\bar{D})>0$.

Proof. - To obtain a contradiction we assume, taking a few subsequences if necessary, that $h_{n}(\bar{D})$ converges to a point set $\left\{x_{\infty}\right\}$. Let $z_{\infty} \in J(G)$ be a repelling periodic point, of period, say $p$, not in the forward orbit of $x_{\infty}$. By the stability of repelling periodic points, there is a continuous map $\mathcal{P}$ from a neighborhood $\mathcal{N}$ of $G$ in $\mathcal{U}_{d}$ to $\overline{\mathbb{C}}$ such that $\mathcal{P}(F)$ is a repelling $p$-periodic point for $F \in \mathcal{N}$ and $\mathcal{P}(G)=z_{\infty}$. Let $z_{n}=\mathcal{P}\left(R_{n}\right)$ for any large $n$ such that $R_{n} \in \mathcal{N}$. As $h_{n}$ conjugates $R$ to $R_{n}$, the point $h_{n}^{-1}\left(z_{n}\right)$ is a repelling $p$-periodic point for $R$. But $R$ has only finitely many such points. So taking again a subsequence if necessary, we may assume $h_{n}^{-1}\left(z_{n}\right)=z$, independent of $n$. As $D \cap J(R) \neq \emptyset$ and preimages of $z$ are dense in $J(R)$, there is $y \in D$ such that $R^{m}(y)=z$ for some $m>0$. Set $y_{n}=h_{n}(y)$. We have $y_{n} \in h_{n}(D) \rightarrow x_{\infty}$, and $R_{n}^{m}\left(y_{n}\right)=z_{n}\left(\right.$ as $\left.R_{n}^{m}\left(y_{n}\right)=R_{n}^{m}\left(h_{n} y\right)=h_{n} R^{m} y=h_{n} z=z_{n}\right)$. Therefore $z_{n} \rightarrow G^{m}\left(x_{\infty}\right)$. On the other hand $z_{n}=\mathcal{P}\left(R_{n}\right) \rightarrow z_{\infty}$. So $z_{\infty}=G^{m}\left(x_{\infty}\right)$. This contradicts the choice of $z_{\infty}$ to be disjoint from the orbit of $x_{\infty}$.

The following result is purely topological, we leave the proof to the reader.

LEMMA 3.2. - Let $h_{n}: S^{2} \rightarrow S^{2}$ be a sequence of homeomorphisms. Let $L \subset S^{2}$ be a compact set having finitely many connected components. Assume that diam ${ }_{\sigma}\left(h_{n}\left(L^{\prime}\right)\right) \rightarrow 0$ for every component $L^{\prime}$ of $L$. If there is one connected component $\Delta$ of $S^{2} \backslash L$ with $\liminf$ diam $_{\sigma} h_{n}(\bar{\Delta})>0$, then for any other component $\Delta^{\prime}$ of $S^{2} \backslash L$, diam $h_{\sigma}\left(\overline{\Delta^{\prime}}\right) \rightarrow 0$.

The following result will be proved in parallel with Theorem A, part (a).2.

Proposition D. - Let $R$ be a rational map of degree $d \geqslant 2$. Let $\gamma^{i}, i=1, \ldots, \nu$, be finitely many $R^{k_{i}}$-invariant connected sets $\left(\right.$ i.e $\left.R^{k_{i}}\left(\gamma^{i}\right) \subset \gamma^{i}\right)$ contained in the Fatou set $F(R)$. Set 
$\Gamma_{0}=\operatorname{closure}\left(\bigcup_{i} \bigcup_{m \geqslant 0} R^{m}\left(\gamma^{i}\right)\right)$ and $\Gamma_{j}=R^{-j} \Gamma_{0}$. Let $R_{n}=h_{n} \circ R \circ h_{n}^{-1}$ be a sequence of rational maps with $h_{n}$ topological conjugacies such that $\max _{i} l_{F\left(R_{n}\right)}\left(h_{n}\left(\gamma^{i}\right), R_{n}^{k_{i}}\right) \rightarrow 0$ as $n \rightarrow \infty$. If $R_{n}$ converges uniformly, then exactly one component $\Delta_{j}$ of $\overline{\mathbb{C}} \backslash \Gamma_{j}$ intersects $J(R)$, and diam $_{\sigma} h_{n}\left(\Delta^{\prime}\right) \rightarrow 0$ for any other component $\Delta^{\prime}$ of $\overline{\mathbb{C}} \backslash \Gamma_{j}$. Consequently, if some $\Gamma_{j}$ separates $J(R)$, the sequence $R_{n}$ has no convergent subsequences.

A quick example for the divergence is a map $R$ with a Herman ring $A$ and a core curve $\gamma$. One can make quasi-conformal deformations of $R$ so that the modulus of $A$ tends to $\infty$ and therefore the hyperbolic length (in particular the dynamical length) of $\gamma$ relative to $A$ tends to 0 . As $\gamma$ is Julia-separating, these deformations do not have convergent subsequences. This case was included in Theorem A.(1) of [5].

Proof of Proposition $D$ and Theorem A, part (a).2. - Set $R_{n}=R_{t_{n}}$ and $h_{n}=\varphi_{t_{n}}$. By assumption $R_{n} \rightrightarrows G$. Fix $j \geqslant 0$. Using the fact that holomorphic maps contract hyperbolic metrics, it is easy to see that the corresponding dynamical length of $h_{n}\left(R^{m} \gamma^{i}\right)$ tends to 0 for any $m$ and $i$. This allows us to apply Proposition $\mathrm{C}$ and the proof of Theorem A, part (a).1, to conclude that $\operatorname{diam}_{\sigma}\left(h_{n}\left(\Gamma^{\prime}\right)\right) \rightarrow 0$ for every component $\Gamma^{\prime}$ of $\Gamma_{j}$. Furthermore $h_{n}\left(\overline{\gamma^{i}}\right)$ accumulates to the set of finitely many $k^{i}$-periodic points of $G$.

Perturbing a repelling periodic point in $J(G)$ of high period we may show that $J(R) \backslash \Gamma_{0} \neq \emptyset$ and therefore $J(R) \backslash \Gamma_{j} \neq \emptyset$.

Fix again any $j \geqslant 0$. We choose a component $\Delta_{j}$ of $\overline{\mathbb{C}} \backslash \Gamma_{j}$ containing points of $J(R)$. By Lemma 3.1, $\liminf _{n \rightarrow \infty} \operatorname{diam}_{\sigma} h_{n}\left(\overline{\Delta_{j}}\right)>0$. So, applying Lemma 3.2, we know that $\operatorname{diam}_{\sigma} h_{n}\left(\overline{\Delta^{\prime}}\right) \rightarrow 0$ for any other component $\Delta^{\prime}$ of $\overline{\mathbb{C}} \backslash \Gamma_{j}$. Applying Lemma 3.1 again, we conclude that $\Delta^{\prime} \cap J(R)=\emptyset$.

This ends the proof of Proposition D.

Assume now $j=0$. Then by Theorem A, part (a).1, for any $\Delta_{0}^{\prime} \neq \Delta_{0}, h_{n}\left(\overline{\Delta_{0}^{\prime}}\right)$ accumulates to the (finite) set of parabolic periodic points of $G$. As a critical point in $\Delta_{0}^{\prime}$ would converge to a critical point of $G$, this is not possible. So critical points of $R$ are contained in $\Delta_{0} \cup \Gamma_{0}$ and therefore in $\Delta_{0}$, as $\Gamma_{0}$ does not contain critical points by definition of admissible set, and the fact that for an admissible pair $(\gamma, U), \bar{\gamma}=\gamma \cup\{\alpha\} \cup\{\beta\}$ with $\alpha$ an attracting (non-superattracting) or parabolic point and $\beta$ a periodic point in $J(R)$ thus non-critical (Lemma 2.2).

\section{Divergence in $\mathcal{V}_{d}$}

Recall that $\pi: \mathcal{U}_{d} \rightarrow \mathcal{V}_{d}$ denotes the canonical projection by the action of Möbius conjugations on $\mathcal{U}_{d}$.

Lemma 4.1. - Let $F_{n}, G \in \mathcal{U}_{d}$. Then $\pi\left(F_{n}\right) \rightarrow \pi(G)$ if and only if there is a sequence of Möbius transformations $H_{n}$ such that $H_{n} \circ F_{n} \circ H_{n}^{-1} \rightrightarrows G$.

Proof. - The sufficiency is due to the fact that $\pi$ is continuous. The necessity is a consequence of the fact that $\pi$ is an open mapping, which can be shown as follows: Let $\mathcal{N} \subset \mathcal{U}_{d}$ be open. Then $H_{*} \mathcal{N}:=\left\{H \circ F \circ H^{-1}, F \in \mathcal{N}\right\}$ is again open, where $H$ is a Möbius map, therefore $\bigcup_{H \text { Möbius }} H_{*}(\mathcal{N})$ is still open. By definition of the quotient topology,

$$
\pi(\mathcal{N})=\pi\left(\bigcup_{H \text { Möbius }} H_{*}(\mathcal{N})\right)
$$

is open in $\mathcal{V}_{d}$. 
Theorem A.(b) now follows from Lemma 4.1 and the fact that part (a) is equally valid if the couple $\left(\varphi_{t}, R_{t}\right)$ is replaced by $\left(H_{t} \circ \varphi_{t}, H_{t} \circ R_{t} \circ H_{t}^{-1}\right)$, for any choices of Möbius maps $H_{t}$.

\section{Admissible pairs, inequalities}

We are going to construct admissible pairs following essentially Petersen [9]. In this entire section we assume that $F$ is a rational map of degree $>1$ with 0 as an attracting or parabolic fixed point and with $\Lambda$ as an immediate attracting basin of 0 .

Recall that $T_{\sigma}$ denotes the translation $z \mapsto z+\sigma$.

\subsection{Attracting case}

Assume that 0 is an attracting (non-super-attracting) fixed point, with $\mu$ as the multiplier (i.e. $\mu=F^{\prime}(0)$ ). Let $\psi: \Lambda \rightarrow \mathbb{C}$ be a linearizing coordinate, i.e. $\psi \circ F=\mu \psi, \psi$ is holomorphic on $\Lambda$ and locally injective at 0 . Denote by $\psi^{-1}$ the local inverse of $\psi$ mapping 0 to 0 , and, by abuse of notation, any analytic extension of it.

Let $c_{1}, \ldots, c_{k}$ denote the critical points for $F$ in $\Lambda$, not in the backwards orbit of 0 . Then the set $\operatorname{Crit}_{\psi, \Lambda}^{\prime}=\left\{\mu^{-n} \psi\left(c_{i}\right) \mid n \geqslant 0,1 \leqslant i \leqslant k\right\}$ consists of the non zero critical values of the (infinite degreed) branched covering $\psi$. For $1 \leqslant i \leqslant k$ let $C_{i}$ denote a logarithm of $\psi\left(c_{i}\right)$ and $M$ a logarithm of $\mu$ (so $\operatorname{Re} M<0$ ). Let $\mathcal{G}$ denote the right half grid

$$
\mathcal{G}:=\left\{n \cdot(-M)+m \cdot 2 \pi i+C_{i} \mid n, m \in \mathbb{Z}, n \geqslant 0,1 \leqslant i \leqslant k\right\} .
$$

So that $z \in \mathcal{G}$ if and only if $\exp (z) \in \mathrm{Crit}_{\psi, \Lambda}^{\prime}$.

Now let us fix a couple $(M, p / q)$ with $M$ a logarithm of $\mu$ and $p / q$ a rotation number (i.e. $q>0,0 \leqslant p \leqslant q-1$ and $p, q$ co-prime). Set $\tau=q M-p \cdot 2 \pi i$. We consider the union $\widehat{\mathcal{G}}$ of straight lines through $\mathcal{G}$ parallel to $\overrightarrow{0 \tau}$. Let $\widetilde{V}$ be an open straight strip in the complement. Then $\left.\psi^{-1} \exp \right|_{\widetilde{V}}$ is univalent and conjugates $T_{\tau}$ to $F^{q}$. We may normalize the dynamical system $\left(\widetilde{V}, T_{\tau}\right)$ to a model system $\left(B_{l_{0}}, T_{1}\right)$ by a conformal affine map $\phi: z \mapsto a+(z / \tau)$.

Let $\tilde{\gamma}$ be the central line of $\widetilde{V}$. Set $\gamma=\psi^{-1} \exp (\tilde{\gamma})$ and $V=\psi^{-1} \exp (\widetilde{V})$. Then $(\gamma, V)$ is an admissible pair with period $q$.

\subsection{Parabolic case}

Assume now that 0 is a parabolic fixed point for $F$. There is a rotation number $p / q$ such that $F^{\prime}(0)=\mathrm{e}^{2 \pi i p / q}$. As $\Lambda$ is an immediate basin of 0 , it has period $q$. A Fatou coordinate is a holomorphic map $\Psi: \Lambda \rightarrow \mathbb{C}$ semi-conjugating $F^{q}$ to $T_{1}$ and injective on a Fatou petal at 0 . As in the attracting case, the critical points of $\Psi$ are preimages of critical points of $F^{q}$, and the critical values of $\Psi$ are contained in a discrete collection of horizontal lines. Let $\widetilde{V}$ be an open horizontal strip in the complement of these lines and let $\tilde{\gamma}$ be its central line. Set $\gamma=\Psi^{-1}(\tilde{\gamma})$ and $V=\Psi^{-1}(\widetilde{V})$ (where $\Psi^{-1}$ is an appropriate extension of the local inverse of $\Psi$ mapping a right half plane into a Fatou petal). Then $(\gamma, V)$ is an admissible pair with period $q$.

\subsection{Inequalities}

Given an annulus $A$, let $m(A)$ be its modulus and $l(A)$ be the hyperbolic length (relative to $A$ ) of its unique closed geodesic. They are both conformal invariants and are related as follows (see McMullen, [6], p. 12):

$$
m(A) \cdot l(A)=\pi
$$


For the model system $\left(B_{l_{0}}, \mathbb{R}, T_{1}\right)$, the quotient surface $B_{l_{0}} / T_{1}$ is an annulus with $\mathbb{R} / T_{1}$ as its unique closed geodesic. We have $l_{B_{l_{0}}}\left(\mathbb{R}, T_{1}\right)=l\left(B_{l_{0}} / T_{1}\right)=l$ and $m\left(B_{l_{0}} / T_{1}\right)=\pi / l_{0}$.

For a triple $(W, \kappa, F)$ conformally conjugate to $\left(B_{l_{0}}, \mathbb{R}, T_{1}\right)$, we have $l_{W}(\kappa, F)=l_{0}$. In the particular case that $W$ is a straight strip and $F$ is a translation $T_{\sigma}$, the set $\kappa$ is automatically the central line of $W$. Denote by $h(W)$ the height of $W$, i.e. the Euclidean distance between the two boundary lines of $W$. Then $l_{W}\left(\kappa, T_{\sigma}\right)=\frac{\pi|\sigma|}{h(W)}$.

Let us go back to the attracting case. We keep the same notation. Let $(M, p / q)$ and $\tau$ be given as above. For $a \in \mathbb{C}$ and the line

$$
L=\{a+t \tau, t \in \mathbb{R}\}=\left\{a+s\left(\frac{p \cdot 2 \pi i}{q}-M\right), s \in \mathbb{R}\right\},
$$

we have $a+\frac{n p \cdot 2 \pi i}{q} \in\left(T_{M}\right)^{n} L$. So $\exp \left(\bigcup_{n \geqslant 0}\left(T_{M}\right)^{n} L\right)$ consists of $q$ disjoint logarithmic spirals (possibly straight lines), and $z \mapsto \mu z$ performs on them a $p / q$ rotation around 0 , since the exponential map semi-conjugates $T_{M}$ to multiplication by $\mu$.

Let $L$ be a line in $\widehat{\mathcal{G}}$ passing through a point of $\log \psi\left(c_{1}\right)$. Let $W$ be the strip between $L$ and $L+2 \pi i$. Then $W-\widehat{\mathcal{G}}$ consists of $k^{\prime} q$ strips $\widetilde{U}_{j}, j=0, \ldots, k^{\prime} q-1$, labelled in their increasing order relative of $i \mathbb{R}$, for some $k^{\prime} \in\{1, \ldots, k\}$. Moreover $T_{M}\left(\widetilde{U}_{j}\right)=\widetilde{U}_{j+k^{\prime} p\left(\bmod k^{\prime} q\right)}$ (modulo $2 \pi i \mathbb{Z})$.

Note that if $k^{\prime}=1$, the height of each $\widetilde{U}_{j}$ is $\frac{2 \pi}{q} \sin \theta$, with $\theta$ being the angle between $\tau$ and $2 \pi i$. This case is realized for example when $\Lambda$ contains only one critical point (i.e. $k=1$ ), or more particularly when $\Lambda$ is a simply connected quadratic basin.

Following Petersen, define $r(M, p / q)=\left|M-\frac{p}{q} 2 \pi i\right| /(2 \sin \theta)$. A geometric interpretation of $r(M, p / q)$ is that it is the radius of the circle through $M$ tangent to the imaginary axis at $2 \pi i \frac{p}{q}$.

Note that $\tau, \widehat{\mathcal{G}}, k^{\prime}, \widetilde{U}_{j}$ and $\theta$ all depend on the choice of $(M, p / q)$.

Define also $r(\mu, p / q)=\inf _{M \in \log \mu} r(M, p / q)$.

The second part of the following is a variant of the Maskit-Yoccoz inequality:

LEMMA 5.1. - 1. A pair $(\gamma, V)$ with $V \subset \Lambda$ is $F^{q}$-admissible if and only if: there is a choice of $(M, p / q)$, two sets $\tilde{\gamma} \subset \widetilde{V} \subset \mathbb{C}$ satisfying:

$\psi^{-1} \exp$ maps $\widetilde{V}$ univalently onto $V, \tilde{\gamma}$ onto $\gamma$;

$\left(T_{n M} \widetilde{V}\right) \cap(\widetilde{V}+2 \pi i \mathbb{Z})=\emptyset$ for $n=1, \ldots, q-1$, and $T_{\tau} \widetilde{V}=\widetilde{V}$;

The quotient $\widetilde{V} / T_{\tau}$ is an annulus with $\tilde{\gamma} / T_{\tau}$ as its core curve.

2. $l_{V}\left(\gamma, F^{q}\right) \geqslant q^{2} r(M, p / q) \geqslant q^{2} r(\mu, p / q) \geqslant q \cdot r\left(\mu^{q}, 0 / 1\right)$, with the first equality realized if and only if $\widetilde{V}$ is a straight strip of height $\frac{2 \pi}{q} \sin \theta$.

Proof. - This is pretty standard material. We will only give a sketch of it. Let $\Omega$ be the lattice in $\mathbb{C}$ generated by $2 \pi i$ and $\log \mu$. Then we have the following Riemann surfaces isomorphisms:

$$
\mathbb{C} / \Omega \stackrel{[\exp ]}{\longrightarrow} \mathbb{C}^{*} / \mu z \stackrel{[\psi]}{\longleftarrow} \Lambda / F
$$

The quotient $[\mathcal{G}]$ consists of finitely many marked points in $\mathbb{C} / \Omega$. By definition of admissible pair $\gamma$ and $V$ descend in $\mathbb{C} / \Omega$ to a simple closed curve $\zeta$ and an annular neighborhood $A$, and then lift to $\tilde{\gamma}$ and $\widetilde{V}$ in $\mathbb{C}$ with a homotopy invariant $\tau \in \Omega$ in the form of $q M-p 2 \pi i$, with $0 \leqslant p \leqslant q-1$. As $V$ and $\widetilde{V}$ are both universal covers of $A$, the map $\psi^{-1}$ exp maps $\widetilde{V}$ univalently onto $V$. The rest of part 1 is just as easy.

Now choose $\widehat{A}$ a fundamental quadrilateral in the lift $\widetilde{A}$, then there is a pair of $\operatorname{arcs}$ in $\partial \widehat{A}$ such that one arc is the translation by $\tau$ of the other. Now the extremal length of the set of arcs joining 
this pair of boundary arcs gives $m(A) \leqslant \frac{\pi}{q^{2} r(M, p / q)}$, with equality if and only if $\widetilde{V}$ is a straight strip of height $\frac{2 \pi}{q} \sin \theta$. As $l_{V}\left(\gamma, F^{q}\right)=l(A)=\pi / m(A)$, we are done.

Therefore if we have a family $\left(V_{t}, \gamma_{t}, F_{t}^{q}\right)$, with $l_{V_{t}}\left(\gamma_{t}, F_{t}^{q}\right) \rightarrow 0$, then $r\left(\mu_{t}^{q}, \frac{0}{1}\right) \rightarrow 0$ and $\mu_{t}^{q} \rightarrow 1$

Our Proposition B is a refinement of Proposition 4.3, [9], there condition 1 is replaced by: $\Lambda_{n}$ is a simply connected quadratic basin, $V_{n}$ lifts to some $\widetilde{U}_{j}$ relative to $\left(M_{n}, p_{n} / q\right)$ so that $r\left(M_{n}, p_{n} / q\right)=\inf _{M \in \log \mu_{n}} r\left(M, p_{n} / q\right)$, and $r\left(\mu_{n}, p_{n} / q\right) \rightarrow 0$. Under these conditions $l_{V_{n}}\left(\gamma_{n}, F_{n}^{q}\right)=q^{2} r\left(\mu_{n}, p_{n} / q\right) \rightarrow 0$.

\section{Relating to Teichmüller theory}

This section is due to a conversation with C. McMullen. I am grateful for his help. The presentation is very close to that of Makienko [5]. See also Pilgrim [11].

Let $R$ be a rational map of degree $d \geqslant 2$. Denote by $\sim$ the grand orbit relation (i.e. $x \sim y$ if there are $n, m \in \mathbb{N}$ such that $R^{n}(x)=R^{m}(y)$ ), by $\Omega^{\text {dis }}(R)$ the union of attracting (nonsuperattracting) and parabolic basins minus the grand orbit of critical points and periodic points, and by $X(R)$ the quotient surface $\Omega^{\text {dis }} / \sim$. By construction the projection $p: \Omega^{\text {dis }} \rightarrow X(R)$ is a covering and thus a hyperbolic isometry. A component of $X(R)$ is either a punctured torus (coming from an attracting basin of $R$ ) or a punctured sphere (coming from a parabolic basin). For any $\zeta \subset X(R)$ denote by $\tilde{\zeta}$ its lift by $p$.

In each punctured torus component $X^{\prime}$ of $X(R)$, there are homotopy classes of simple closed curves corresponding to the quotients of loops around the attracting point. Every other simple closed curve $\zeta$ in $X(R)$ lifts to grand orbits of periodic open arcs, and, in case $\zeta$ is a geodesic, it has a collar neighborhood $A$ such that $(\tilde{\zeta}, \widetilde{A})$ is the grand orbit of an admissible pair.

The Teichmüller space of $R$ consists of, up to isotopy, pairs $(\phi, F)$ such that $\phi$ is a quasiconformal conjugacy from $R$ to $F$. See McMullen and Sullivan, [7] for details, where it is proved that Teich $(R) \approx \operatorname{Teich} X(R) \times$ something else. By abuse of notation, denote by $\pi$ the natural projection from $\operatorname{Teich}(R)$ into $\mathcal{V}_{d}$. A problem coming from the analogy with 3-manifolds is: when is $\overline{\pi(\operatorname{Teich}(R))}$ compact?

Following Pilgrim [11], we make the following

DEFINITION (Cylinders). - We say that a geodesic multicurve $\zeta \subset X(R)$ (union of finitely many disjoint simple closed geodesics) represents a cylinder if some $\Gamma_{j}$ separates $J(R)$, where $\Gamma_{j}=R^{-j}\left(\Gamma_{0}\right)$ and $\Gamma_{0}$ is the closure of the periodic $\operatorname{arcs}$ in $\tilde{\zeta}$. We say that $R$ is cylindrical if such a multicurve exists.

(For intuition about cylinders and how they occur in the study of Kleinian groups, see [11]).

To each geodesic multicurve $\zeta$ and a pair $(\phi, F)$ as above, there corresponds a unique geodesic multicurve $\phi_{*}(\zeta)$ in $X(F)$ isotopic to the quotient of $\phi(\tilde{\zeta})$. There is a natural mapping $L_{\zeta}: \operatorname{Teich}(R) \rightarrow \mathbb{R}^{+}$such that $L_{\zeta}([\phi, F])$ is the maximum of the hyperbolic lengths of the geodesics in $\phi_{*}(\zeta)$. Our results can be now interpreted as follows:

THEOREM E. -If $\zeta$ represents a cylinder for $R$, and $\left[\phi_{n}, R_{n}\right] \in \operatorname{Teich}(R)$ with $L_{\zeta}\left(\left[\phi_{n}, R_{n}\right]\right) \rightarrow 0$ then $\pi\left(R_{n}\right) \rightarrow \infty$ in $\mathcal{V}_{d}$

Proof. - To obtain a contradiction, we assume, taking a subsequence if necessary, $\pi\left(R_{n}\right) \rightarrow \pi(G)$. By Lemma 4.1 there are representatives $R_{n}$ and $G$ such that $R_{n} \rightrightarrows G$. Recall that for $\gamma \subset \Omega^{\text {dis }} \subset F(R)$ a connected $R^{k}$-invariant set, the dynamical length is defined to be $l_{\Omega^{\text {dis }}}\left(\gamma, R^{k}\right)=\sup _{z \in \gamma} d_{\Omega^{\text {dis }}}\left(z, R^{k}(z)\right)$. 
For $\gamma$ a periodic arc in $\tilde{\zeta}$, of period say $k$, it is also a geodesic in $\Omega^{\text {dis }}$. So, denoting by $\kappa_{z}$ the sub-arc of $\gamma$ between a point $z$ and $R^{k}(z)$, we have

$$
l_{F(R)}\left(\gamma, R^{k}\right) \leqslant l_{\Omega^{\mathrm{dis}}}\left(\gamma, R^{k}\right) \leqslant \sup _{z \in \gamma} \text { ength }_{\Omega^{\mathrm{dis}}}\left(\kappa_{z}\right)=\operatorname{length}_{X(R)}(p(\gamma)) \leqslant L_{\zeta}([I d, R]) .
$$

Similarly for any periodic arc $\gamma_{n}$ in the lifts of $\left(\phi_{n}\right)_{*}(\zeta), l_{F\left(R_{n}\right)}\left(\gamma_{n}, R_{n}^{k}\right) \leqslant L_{\zeta}\left(\left[\phi_{n}, R_{n}\right]\right)$.

By assumption $L_{\zeta}\left(\left[\phi_{n}, R_{n}\right]\right) \rightarrow 0$ as $n \rightarrow \infty$. We can then apply Proposition $\mathrm{C}$ to conclude that $\operatorname{diam}_{\sigma}\left(\overline{\gamma_{n}}\right) \rightarrow 0$. We can then apply Proposition D to conclude that, for $\Gamma_{0}$ the closure of the union of the periodic $\operatorname{arcs}$ in $\tilde{\zeta}$, and $\Gamma_{j}=R^{-j}\left(\Gamma_{0}\right)$, there is exactly one component $\Delta_{j}$ of $\overline{\mathbb{C}} \backslash \Gamma_{j}$ intersecting $J(R)$. This contradicts the assumption that $\zeta$ represents a cylinder.

COROLlARY F. - If $R$ is cylindrical then $\overline{\pi(T e i c h(f))}$ is not compact.

Proof. - Our pinching deformations related to a collar neighborhood of $\zeta$ provide a path $\left[\varphi_{t}, R_{t}\right] \in \operatorname{Teich}(R)$ with $L_{\zeta}\left(\left[\varphi_{t}, R_{t}\right]\right) \rightarrow 0$ as $t \rightarrow 1$ (see Remark 1 ).

\section{Examples and applications}

It is known that for $f_{n}$ and $g$ rational maps, $f_{n} \rightrightarrows g$ if and only if there are choices of coefficients of $f_{n}$ tending to that of $g$ and $g$ has the same degree as $f_{n}$ for $n$ large, in other words, the uniform convergence topology on $\mathcal{U}_{d}$ coincides with the algebraic convergence topology. See for example Beardon [1], §2.8. The constructions of the following examples are guided by the idea of perturbing postcritically finite maps and by Pilgrim-Tan's arc-blowing technique. See Makienko [5], Pilgrim [11] and Pilgrim-Tan [12,13] for more theoretical treatments. The figures are drawn by K. Pilgrim.

\subsection{Examples of convergence}

Example 1. $-f_{c_{0}}: z \mapsto z^{2}+c_{0}$ with $c_{0}=3 / 16<1 / 4$. Let $\left.\gamma=\right] \alpha, \beta[$ be the real segment between the two fixed points and $U$ a suitable neighborhood of $\gamma$, symmetric with respect to $\mathbb{R}$ and invariant by $f_{c_{0}}$. Then $(\gamma, U)$ is an admissible pair. For $c \in\left[c_{0}, 1 / 4\left[\right.\right.$, the corresponding $f_{c}$ can be regarded as a path of pinching deformations of $f_{c_{0}}$ along $(\gamma, U)$. This path converges to $f_{1 / 4}$ as $c \rightarrow 1 / 4$, with $\overline{\gamma_{c}} \rightarrow 1 / 2$. See Fig. 20 in Milnor [8], p. 113 .

Example 2. - This is an example where maps have symmetries and, if the pinching maps $\varphi_{t}$ are not normalized properly, $\varphi_{t}(\alpha)$ may not converge, but $R_{t}$ converges. Let

$$
f(z)=\frac{1}{1-s^{2}}\left(z^{5}-\frac{10}{3} z^{3}+5\left(1-s^{2}\right) z\right), \quad \text { with } f^{\prime}(z)=\frac{5}{1-s^{2}}\left(\left(z^{2}-1\right)^{2}-s^{2}\right) .
$$

For $s>0$ and $c=\sqrt{1+s}$ the largest critical point, $f(c)=\frac{c^{3}}{1-s^{2}}\left(3-\frac{1}{3}-4 s\right)$. So $f(c)=c$ if and only if $s=5 / 9$. From this one can deduce that for $s=\frac{5}{9}-\varepsilon$ the map $f$ has four real critical points $\pm c, \pm c^{\prime}$ and five real fixed points $\pm \beta, \pm \alpha, 0$ positioned in the following order:

$$
-\beta<-\alpha<-c<-c^{\prime}<0<c^{\prime}<c<\alpha<\beta
$$

and that $\alpha_{ \pm}$are attracting. Now pinching simultaneously $]-\beta,-\alpha[$ and $] \alpha, \beta[$ we get a converging sequence of polynomials. However, for $\varphi_{t}$ a path of conjugacies, normalized so that $\varphi_{t}(0)=0$ and $\varphi_{t}( \pm \alpha)= \pm 1$, we can replace $\varphi_{t}$ by $-\varphi_{t}$ for a sequence $t_{n} \rightarrow 1$. Then 


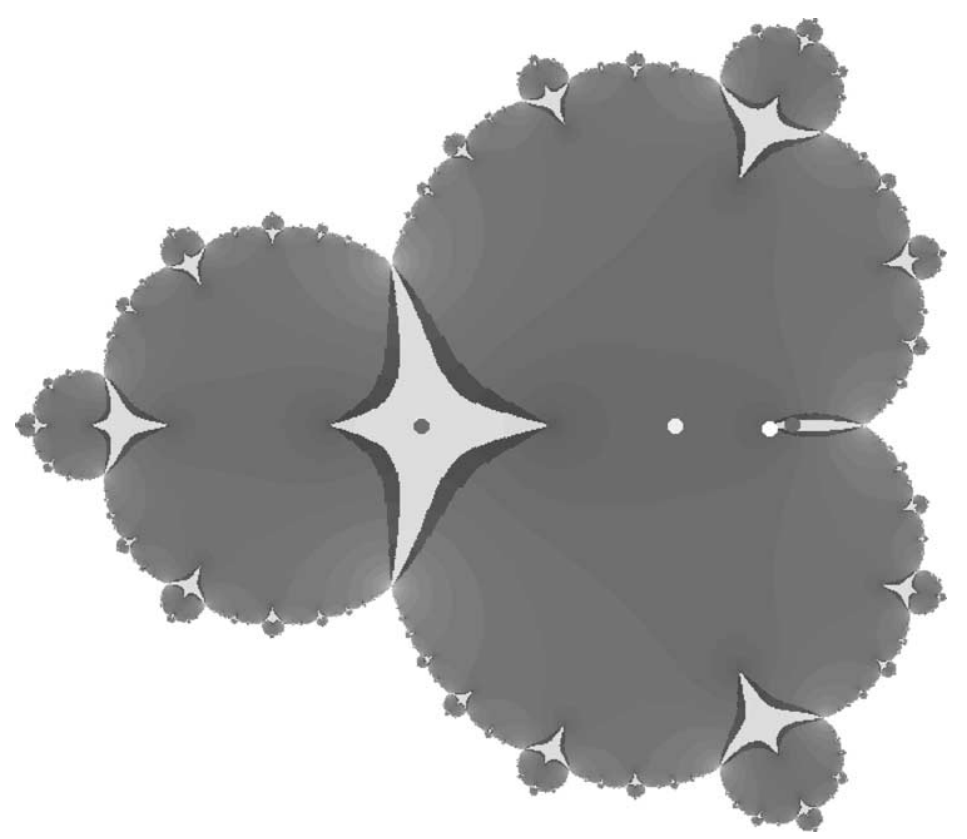

Fig. 1. Example $3^{\prime}$. The dark regions are grand orbits of neighborhood of $\Gamma_{0}$. In the limit of pinching, these regions collapse, forcing the uniform light gray regions to collapse as well. Observe that the largest of such regions contains the critical point $c_{2}$ shown in medium gray whose image $f\left(c_{2}\right)$ (shown in the same color) is just to the right of the attractor 0 (the white dot to the right). The other critical point $c_{1}$ is shown in very light gray.

the images of $\alpha$ under this new family of conjugacies oscillate between -1 and 1 and therefore do not converge.

Example 3. - This is a case where $\gamma$ contains a critical value. Let $f$ be the cubic polynomial $z^{3}+1.6 z^{2}+0.5 \overline{6} z$. It has the following property: The fixed points $0, \beta, \beta^{\prime}$ and the critical points $c_{1}, c_{2}$ are all real with $\beta^{\prime}<c_{2}<c_{1}<0<f\left(c_{2}\right)<\beta$, and $J(f)$ is a Jordan curve. Take $\left.\gamma=\right] 0, \beta[$.

Example $3^{\prime}$. - This is a convergence case where $\overline{\mathbb{C}} \backslash \Gamma_{0}$ has a component $\Delta_{0}^{\prime}$ not intersecting the Julia set. Take $f$ as in Example 3. Take $\gamma_{1}, \gamma_{2}$ two boundary arcs of a neighborhood of ] $0, \beta\left[\right.$. Then there are appropriate neighborhoods $U_{1}, U_{2}$ such that $\left(g_{1}, U_{1}\right),\left(\gamma_{2}, U_{2}\right)$ forms an admissible set. Set $\Gamma_{0}=\overline{\gamma_{1} \cup \gamma_{2}}$. The pinching deformations converge, with a component $\Delta^{\prime}$ of $\overline{\mathbb{C}} \backslash \Gamma_{0}$ containing a critical value, but containing no Julia points nor critical points. The diameter of $\Delta^{\prime}$ tends to 0 under pinching. This is also an example that some critical point might be pushed into the Julia set under pinching and become eventually parabolic.

Example 4. - Pinching in a parabolic basin. Let $F(z)=z^{3}+z^{2}+z$. Then $\left.\gamma=\right]-1,0[$ is a pinchable arc contained in the immediate basin of the parabolic fixed point 0 which has only one attracting petal. The pinching along $\gamma$ converges, and the limit map has a parabolic fixed point with two fixed attracting petals instead.

Example 5. - Fat basilica with a cauliflower attached at the beta fixed point. A case where two different types of parabolic orbits develop at the limit, and one of them is due to pinching. Let $g_{b}(z)=-z^{3}+2 b \sqrt{b+1} z^{2}-b z$. One can check easily that for $b=1-\varepsilon, g_{b}$ has 0 as the unique real fixed point and two real critical points; but $g_{1}$ has two real fixed points 0 and $\sqrt{2}$ and two real critical points. 

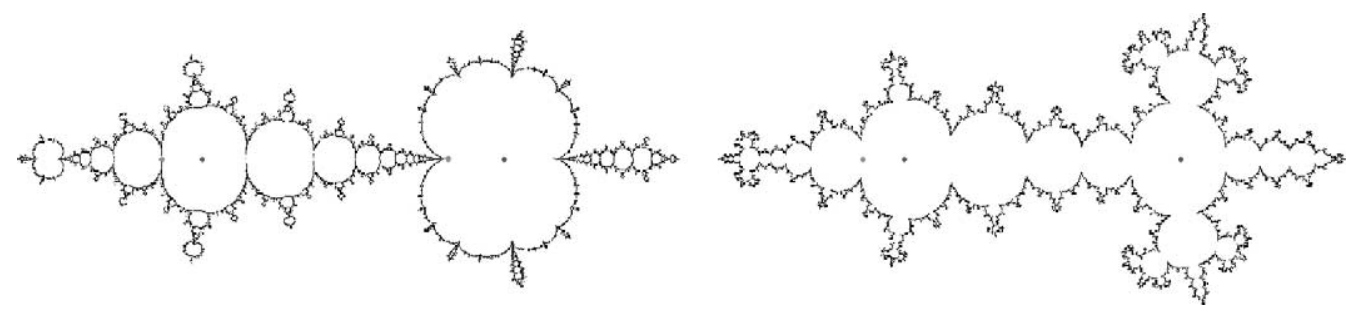

Fig. 2. Example 5, with $b=1$ and $b=0.95$.
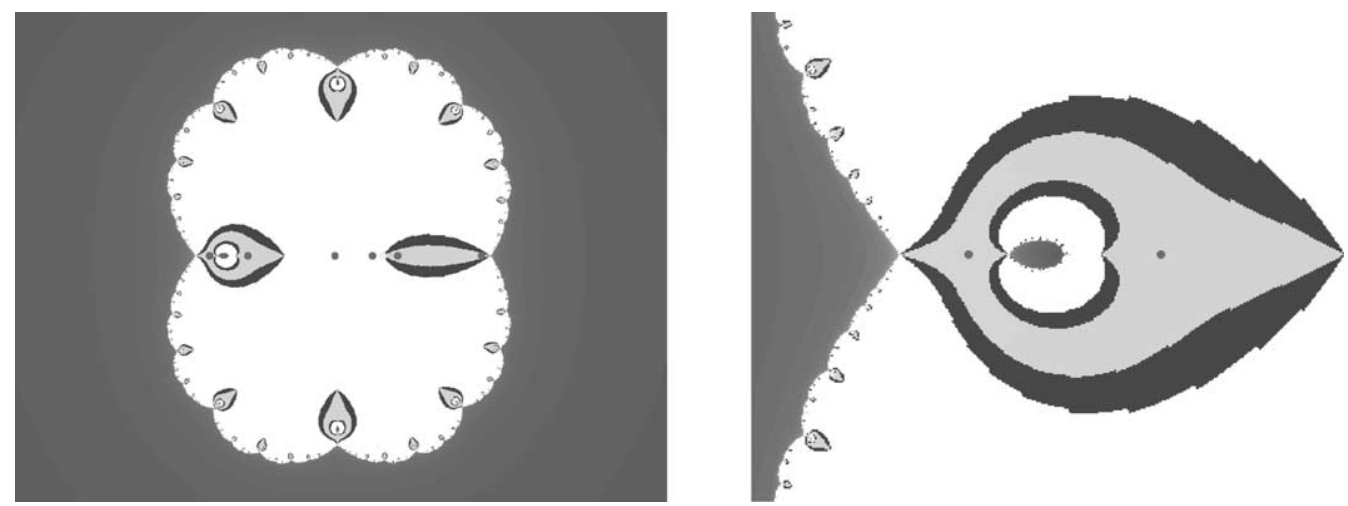

Fig. 3. Example $6^{\prime}$ and zoom.

\subsection{Examples of divergence}

A. $\Gamma_{0}$ is not Julia-separating, but some $\Gamma_{j}, j>0$, is:

Example 6. - Let

$$
R(z)=z^{2}+\frac{3}{16}-\frac{u}{z+\frac{\sqrt{5}}{4}}, \quad u=0.01 .
$$

For $u=0$, this is just a quadratic polynomial with two fixed points $1 / 4,3 / 4$ and maps $\sqrt{5} / 4$ to the middle point $1 / 2$ of them. By stability of hyperbolic fixed points and symmetry $R$ has two real fixed points $\alpha, \beta$ close to the above ones. The arc $\gamma=] \alpha, \beta[$ is forward invariant and is part of an admissible pair. Moreover $\gamma$ contains two critical values and $R^{-1} \gamma$ contains a Jordan curve $L$ disjoint from $J(R)$ and separating $J(R)$. The Julia set of $R$ is disconnected. In this case $\pi\left(R_{t}\right) \rightarrow \infty$ in $\mathcal{V}_{3}$ (we have $R \in W_{1}$ in the notation of [5]).

Example 6'. - Take $R$ as in Example 6, and $\gamma_{1}, \gamma_{2}$ the two boundary components of $U$. One can define pinching along $\bar{\gamma}_{1} \cup \bar{\gamma}_{2}$. It forms a Jordan curve not separating the Julia set. But its preimage consists of itself together with two Julia-separating Jordan curves.

Example 7 ( $\Gamma_{j}$ has a separating curve in a preperiodic component). - Take $f_{c_{1}}$ with $-1<$ $c_{1}<-3 / 4$ so that it has a period two attracting cycle. As in Example $1, f_{c}$ is a converging path, for $c_{1} \leqslant c<-3 / 4$ and $c \rightarrow-3 / 4$. But in this case the Fatou set has infinitely many components. And one can make similar perturbations as in Example 6 in either periodic or non-periodic components to get many diverging paths of pinching deformations. 

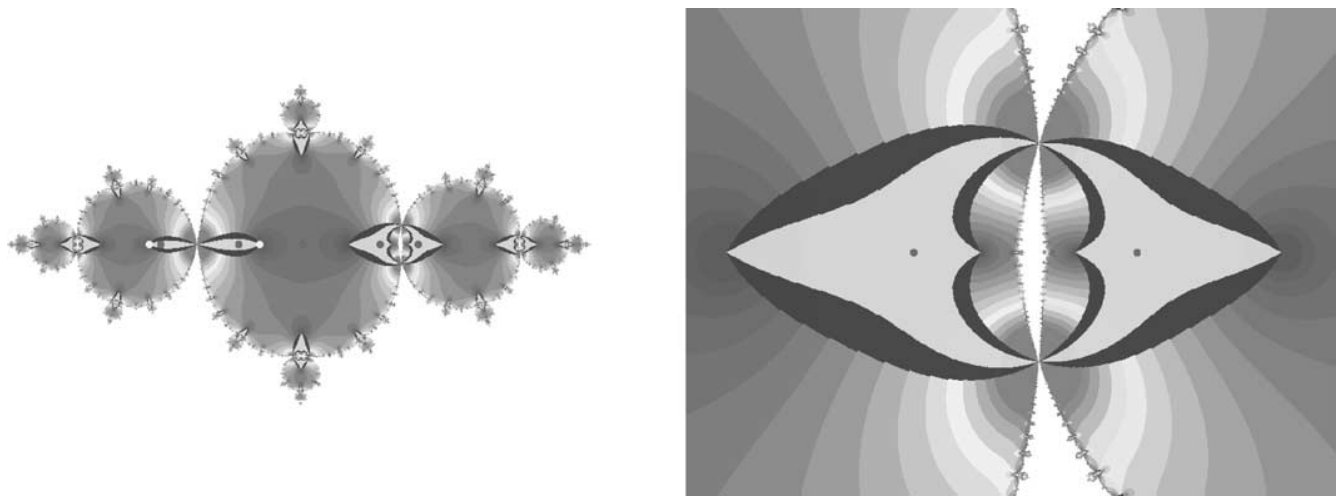

Fig. 4. Example 8 and zoom.

Example 8 (The postcritically finite version of this map was first obtained by K. Pilgrim. See [11], pp. 70-72). - This is similar to Example 6 but with connected Julia set.

$$
F(z)=z^{2}-\frac{13}{16}+\frac{v}{z+\frac{2-\sqrt{17}}{4}}, \quad v=0.01 .
$$

For $v=0$, the map has a repelling fixed point $\alpha=(2-\sqrt{17}) / 4$ and an attracting periodic cycle $a, b$ with $\gamma=] a, \alpha\left[\right.$ a pinchable arc of period two. In this case $\Gamma_{0}:=\bar{\gamma} \cup \overline{F(\gamma)}=[a, b]$. For $v>0$ small, this set is stable, but the map has a pole at $-\alpha$. And $v=0.01$ is chosen so that $\Gamma_{0}$ has two critical values, one in $] a, \alpha[$ and one in $] \alpha, b\left[\right.$. As a consequence $\Gamma_{1}=F^{-1} \Gamma_{0}$ contains a Julia separating Jordan curve.

\section{B. $\Gamma_{0}$ is Julia-separating:}

Example 9 ( $\Gamma_{0}$ touches only one Fatou component, is formed by one single orbit of arcs, i.e. lifts of one closed geodesic in the quotient surface). - Take $f_{-1}: z \mapsto z^{2}-1$ and perturb it into $R(z)=\left(z^{2}-1\right) /\left(\frac{8}{9} z+1\right)$ so that $\infty$ becomes attracting but not superattracting. For the rotation number $1 / 2$, we may construct period-two arcs issuing from $\infty$ as in $\S 5$ : as the basin of $\infty$ is quadratic and simply connected, $k^{\prime}=1$ and there are exactly two strips $\widetilde{U}_{1}$ and $\widetilde{U}_{2}$ in any $W$ as in $\S 5$. Their central lines project to two arcs issuing from $\infty$ and are permuted by the new map. These two arcs must land on a common fixed point on the Julia set (as they land either on a period two cycle or on a fixed point, but $R$ has a unique period two cycle which is super-attracting). So their union is a Jordan curve separating the Julia set. Any pinching along them is divergent ( $R \in W_{2}$ in the sense of [5]). The maps $R(z)$ and $f_{-1}(z)$ are qc-conjugate on neighborhoods of Julia sets. See Fig. 9 in Milnor [8], p. 89 for the Julia set of $f_{-1}$.

Example 10 ( $\Gamma_{0}$ touches only one Fatou component, is formed by lifts of two closed quotient geodesics).-

$$
f(z)=\frac{z^{3}}{1+\frac{3}{2} z^{2}}+a z
$$

with $a=0.01$. For $a=0$ this map is conjugate (by $1 / z$ ) to the cubic polynomial $z^{3}+\frac{3}{2} z$ which has two fixed simple critical points and a double critical point at $\infty$. The perturbation is chosen so that 0 for $f$ is only attracting and $\mathbb{R}$ is in its basin consisting of two invariant arcs of rotation number $0 / 1$. So $\Gamma_{0}=\mathbb{R} \cup\{\infty\}$ is a Julia-separating Jordan curve ( $f \in W_{2}$ in the sense of [5]). 


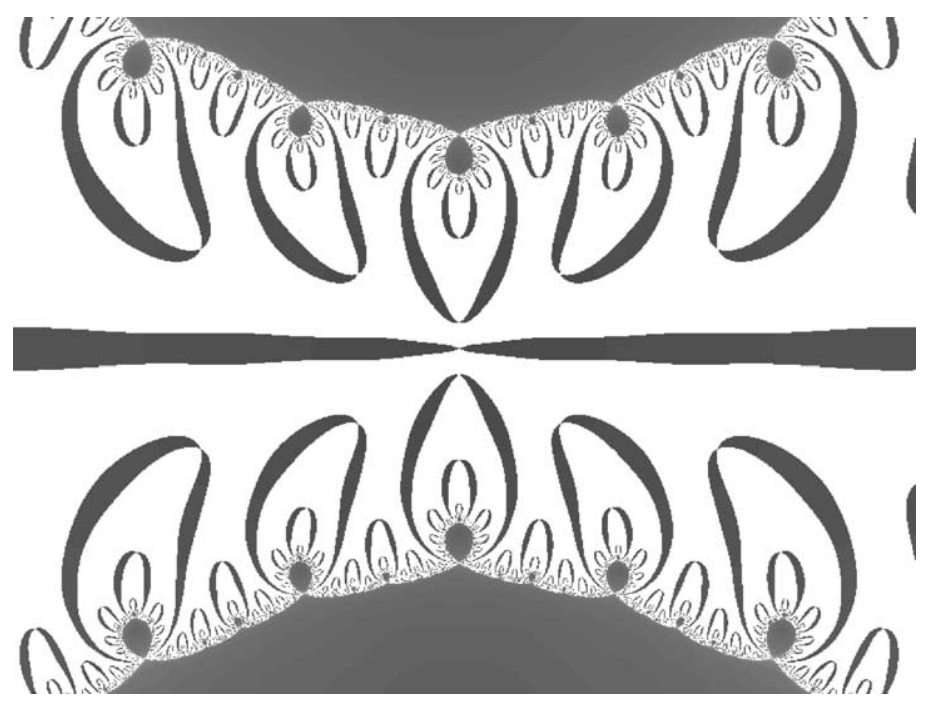

Fig. 5. Example 10.

Example 11 ( $\Gamma_{0}$ touches two Fatou components). - Relaxed cubic Newton's method (see Tan $[14,15]) R(z)=z-h \frac{P(z)}{P^{\prime}(z)}$, where $P$ is a cubic polynomial with simple roots and $0<h \leqslant 1$. When $h=1$ two of the three superattracting fixed basins (of roots of $P$ ) have many shared periodic points on the boundary, as shown in [14]. $R_{h}$ is a perturbation so that the superattracting fixed points become only attracting (of multiplier $1-h$ ). For suitably chosen $P$ these basins are of degree 2 ; in particular their boundary contains a unique orbit of given rotation number. Then as in Example 9 one can show that the projection of suitable strips and their central lines should land at the perturbed periodic points, giving invariant Jordan curves separating the Julia set (in $W_{3}$ in the sense of [5]).

Example 12 ( $\Gamma_{0}$ touches more than two Fatou components and is minimal). -

$$
Q(z)=\frac{8}{3} \cdot \frac{z^{5}}{1-\frac{10}{3} z^{2}+5 z^{4}}+a z
$$

with $a=0.25$. For $a=0$ this is conjugate (by $1 / z$ ) to a degree five polynomial with two fixed points each of type $z^{3}$. After perturbation, $\mathbb{R} \cup\{\infty\}$ is invariant, containing three attracting fixed points, three repelling fixed points and the complement consists of six intervals each is an invariant pinchable arc. Let $\Gamma_{0}=\mathbb{R} \cup\{\infty\}$. It is a Jordan curve running through three Fatou components and is Julia separating. The pinching deformations along $\Gamma_{0}$ diverge. This example can be generalized to construct maps such that $\Gamma_{0}$ runs through $N$ Fatou components for any given number $N \geqslant 3$.

Application. For $h(z)=z^{3}+\varepsilon$ a small perturbation of $z^{3}$, the repelling cycle $\{a, b\}$ with external angles $1 / 4,3 / 4$ can never be pinched. We may prove this by contradiction: denote by $\alpha$ the attracting fixed point. Assume that there is a simple closed geodesic in the quotient surface of rotation number $1 / 2$ whose lift contains a periodic-two cycle of arcs joining $\alpha$ to $a$ and $b$. Then one can define a path of pinching deformations along these arcs and obtain a path $h_{t}$ of cubic polynomials, qc-conjugate to $h$. The set of cubic polynomials with connected Julia set is compact. Hence $h_{t_{n}}$ converges to a cubic polynomial $g$ for some sequence $t_{n} \rightarrow 1$. The map $g$ 


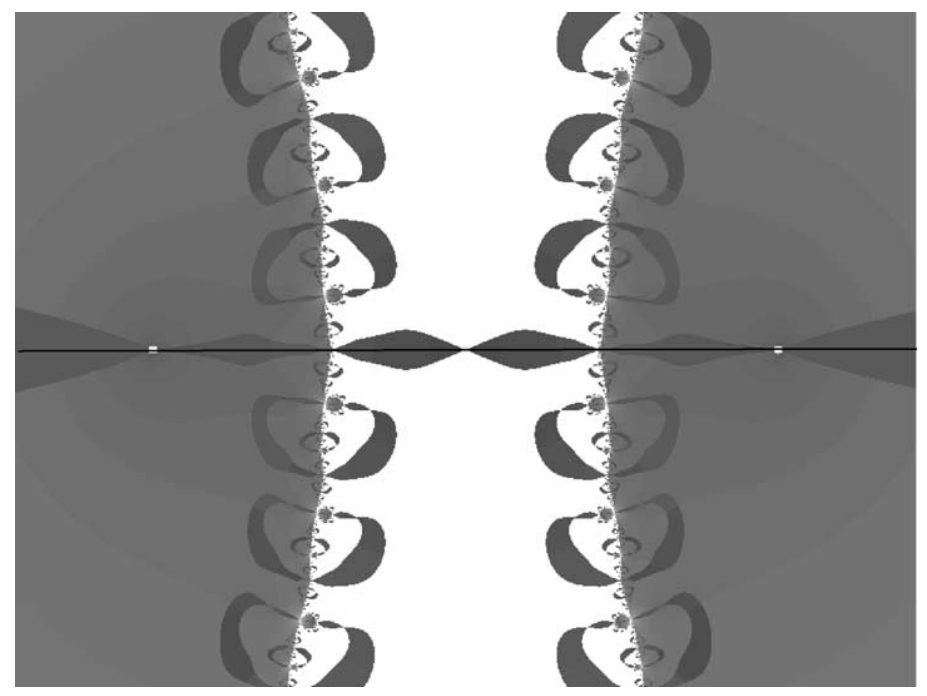

Fig. 6. Example 12.

has a unique critical point of multiplicity 2 , just as the maps $h_{t}$ do. By Theorem A, for the map $g$ the two external rays of angles $1 / 4,3 / 4$ land at a common fixed point. Therefore the union of these two rays together with the landing point forms a line separating $\mathbb{C}$ into two parts $C^{\prime}, C^{\prime \prime}$. By looking at the angle tripling map one may conclude that $g$ has a critical point in each $C^{\prime}, C^{\prime \prime}$. This contradicts the fact that $g$ has a unique critical point.

\section{Acknowledgements}

My first thanks go to the participants of our working seminar at ENS Paris, for motivating this research. I am grateful to G. Cui, C. McMullen, C. Petersen and K. Pilgrim for their very helpful comments on preliminary versions of the paper. More particularly, McMullen pointed out an alternative proof Lemma 2.1 which allowed me to establish Proposition C and to put Theorem A in a more general form. The figures are kindly computed by Pilgrim, using a program of Dan Sørensen.

\section{Appendix A. Relating to the paper [5]}

To avoid confusion statements in italic refer to results in [5].

Part (b) of our Theorem A is a generalization of Theorem A, Part (2) in the following sense: we deal with topological conjugacies shrinking appropriate dynamical lengths, with quasi-conformal pinching deformations as a particular case; we allow the pinchable arcs $\gamma$ to run through forward orbits of critical points; and finally we allow some preperiodic part of the pinching (i.e. $\Gamma_{j}$ for $j>0$ ) to separate $J(R)$, whereas the periodic part (i.e. $\Gamma_{0}$ ) may or may not separate $J(R)$. We provide a proof that does not use the Maskit inequality. Also, Part (a).2 treats the case that $\overline{\mathbb{C}} \backslash \Gamma_{j}$ may have components disjoint from the Julia set, a case that is missing in Proposition 1.1.

Regarding the proofs, the original proof of Theorem A, Part (2) relies on two intermediate results Lemma 1.2 and Proposition 1.1 + Theorem 1.1, whose proofs seem incomplete (see below). Our Lemma 3.1 replaces Lemma 1.2, and Lemma 2.1 by passes Proposition $1.1+$ Theorem 1.1 and proves a consequence of them directly (see the remark after Theorem 1.1, p. 13, 
top). Lemma 1.2 is also used elsewhere in [5], for example in the proof of Theorem A, Part (1). Its replacement Lemma 3.1 is equally suitable at these places.

As for the original proof of Lemma 1.2, in Part (2) it states the equal cardinality of two finite sets, but the proof gives only an injection from one set to the other, and the constant $N_{R}$ chosen in the proof is certainly too small (the statement is however true, as shown recently by C. McMullen).

The statement of Proposition 1.1 is not correct as shown by our Example 3. This is due to the fact that the assertion 'each $D_{j}$ must contain a critical point' in the proof is not always true.

\section{REFERENCES}

[1] BEARDON A., Iteration of Rational Functions, Springer-Verlag, 1991.

[2] CUI G., Geometrically finite rational maps with given combinatorics, preprint, February 1997.

[3] Cui G., Deformations of geometrically finite rational maps, April 1997.

[4] HAÏSSINSKY P., Pincement de polynômes, to appear.

[5] MAKIENKO P., Unbounded components in parameter space of rational maps, in: Conformal Geometry and Dynamics, Vol. 4, 2000, pp. 1-21.

[6] McMullen C., Complex Dynamics and Renormalization, Ann. Math. Studies, Vol. 135, Princeton Univ. Press, 1994.

[7] MCMullen C., Sullivan D., Quasiconformal homeomorphisms and dynamics III: The Teichmüller space of a holomorphic dynamical system, Adv. Math. 135 (1998) 351-395.

[8] Milnor J., Dynamics in One Complex Variable: Introductory Lectures, Vieweg, 1999.

[9] Petersen C., No elliptic limits for quadratic maps, Ergodic Theory Dynamical Systems 19 (1999) $127-141$.

[10] Petersen C., On the Pommerenke-Levin-Yoccoz inequality, Ergodic Theory Dynamical Systems 13 (1993) 785-806.

[11] Pilgrim K., Cylinders for iterated rational maps, Ph.D. Thesis, University of California, Berkeley, 1994.

[12] Pilgrim K., TAN L., Combining rational maps and controlling obstructions, Ergodic Theory Dynamical Systems 18 (1998) 221-246.

[13] PILgRim K., TAN L., On disc-annulus surgery of rational maps, in: Jiang Y., Wen L. (Eds.), Proceedings of the International Conference in Dynamical Systems in Honor of Professor Liao Shan-tao 1998, World Scientific, 1999, pp. 237-250.

[14] TAN L., Branched coverings and cubic Newton maps, Fundamenta Mathematicae 154 (1997) 207260.

[15] TAN L., Continuous and discrete Newton's algorithms, in: Proceedings of the Conference on Complex Analysis, Nankai Institute of Mathematics, 1992, International Press, 1994, pp. 208-219.

Lei TAN

Unité CNRS-UPRESA 8088,

Département de Mathématiques,

Université de Cergy-Pontoise, 2

Avenue Adolphe Chauvin,

95302 Cergy-Pontoise cedex, France

E-mail: tanlei@math.u-cergy.fr 